\section{RADIATION SUB-STUDY TO CHARACTERIZE SAFETY AND TOLERABILITY OF LOW-DOSE RADIATION IN COMBINATION WITH AFAMI-CEL IN PATIENTS WITH ADVANCED CANCERS (NCT03132922)}

'James Welsh*, 'Danxia Ke, ${ }^{1}$ Nahum Puebla Osorio, 'Hampartsoum Barsoumian, ${ }^{2}$ Bryan Jackson, ${ }^{2}$ Jane Bai, ${ }^{2}$ Marisa Rosenberg, ${ }^{2}$ Cheryl McAlpine, ${ }^{2}$ Robyn Broad, ${ }^{2}$ Ashley Liddle, ${ }^{2}$ Jean-Marc Navenot, ${ }^{2}$ Stavros Rafail, ${ }^{2}$ Ruoxi Wang, ${ }^{2}$ Amy Sauer, ${ }^{2}$ Quan Lin, ${ }^{2}$ Hassan Danesi, 'David Hong. ${ }^{1}$ The University of Texas MD Anderson Cancer Center, Houston, TX, USA; '2Adaptimmune, Philadelphia, PA, USA

Background Autologous cell therapies with an engineered Tcell receptor targeting MAGE-A4 have shown responses in patients with synovial sarcoma ${ }^{1}$ with additional responses in myxoid/round cell liposarcoma (MRCLS), head and neck, lung, esophagogastric junction, and melanoma cancers. ${ }^{2} 3$ Low-dose radiation may control tumor growth locally and modulate stroma of solid tumors, ${ }^{4}$ potentially facilitating T-cell infiltration into tumors and antitumor activity.

Methods Sub-study designed to assess safety, tolerability, and efficacy in up to 10 patients with low-dose radiation in combination with lymphodepleting chemotherapy, followed by afami-cel (an autologous TCR cell T-cell therapy targeting MAGE-A4). Eligible patients are HLA-A*02^ + with MAGEA4 expressing tumors including urothelial, melanoma, head and neck, ovarian, non-small cell lung, esophageal, gastric, synovial sarcoma, and MRCLS cancers. Patients receive afamicel by infusion following low-dose radiation and lymphodepleting chemotherapy. Radiation was 4.2-7 Gy per lesion or isocenter (maximum of 5). Lymphodepleting regimen was IV fludarabine $30 \mathrm{mg} / \mathrm{m}^{\wedge}$ 2/day for 4 days ( -7 to -4$)$ and cyclophosphamide $600 \mathrm{mg} / \mathrm{m}^{\wedge}$ 2/day for 3 days $(-7$ to -5$)$. Afami-cel doses ranged from $1.2 \times 10^{\wedge} 9$ to $10 \times 10^{\wedge} 9$ transduced cells. Pts receive afami-cel infusion on Day 1.

Results As of Dec 27, 2020, a total of 8 patients, including 4 patients (1 male) with melanoma (2), HNSCC (1), or ovarian (1) cancers received low-dose radiation and afami-cel. Most frequently reported AEs (4/4 pts) were leukopenia/decreased white blood cell count, lymphopenia/decreased lymphocyte count, and neutropenia/decreased neutrophil count; all of which were related to the lymphodepletion regimen. The most commonly ( $>1$ patient) reported AEs considered related to Tcell infusion were cytokine release syndrome (2/4 pts) and fatigue $(2 / 4$ pts). Two patients had a total of 5 SAEs: adrenal insufficiency, hyperglycemia, neurotoxicity, pneumonia aspiration, and pneumothorax. The only SAE considered to be related to treatment was Grade 3 neurotoxicity. Best overall responses per RECIST 1.1: 1 partial response (melanoma, $-42 \%$ in target lesions), 2 stable diseases (ovarian cancer, $-23 \%$; HNSCC, no change), and 1 patient did not have post-baseline scans yet. Translational analyses showed peripheral persistence and serum cytokine response profiles consistent with that of afami-cel monotherapy, whilst a relatively greater $\mathrm{T}$ cell infiltration in tumor biopsies was evident.

Conclusions Afami-cel with low-dose radiation has had an acceptable safety profile. Most AEs were consistent with those typically experienced by cancer patients undergoing lymphodepletion cytotoxic chemotherapy and cellular therapy. Infused T-cells were observed in tumor biopsies at high frequency, and one patient exhibited a clinical partial response.

Trial Registration NCT03132922

\section{REFERENCES}

1. . Van Tine BA, et al. CTOS 2020.

2.. Hong DS, et al. ASCO 2020

3.. Hong DS, et al. SITC 2020.

4.. De Selm C, et al. Mol Ther 2018;26(11):2542-2552.

http://dx.doi.org/10.1136/jitc-2021-SITC2021.376 